\title{
ANÁLISE ECONOMÉTRICA DO PREÇO EXTERNO DO COMPENSADO BRASILEIRO
}

\author{
Alexandre Nascimento de Almeida ${ }^{1}$, Humberto Ângelo ${ }^{1}$, João Carlos Garzel Leodoro da Silva ${ }^{2}$ \\ ${ }^{1}$ Eng. Florestal, Dr., Depto. de Engenharia Florestal, UnB, Brasília, DF, Brasil - alexfloresta@pop.com.br; humb@unb.br \\ ${ }^{2}$ Eng. Florestal, Dr., Depto. de Economia Rural e Extensão, UFPR, Curitiba, PR, Brasil - garzel@ufpr.br
}

Recebido para publicação: 16/07/2009 - Aceito para publicação: 01/03/2010

\begin{abstract}
Resumo
Este trabalho analisa o preço internacional do compensado brasileiro. Em especial, estima as elasticidades das principais variáveis que afetam a sua formação. Como base metodológica, utilizouse o método dos Mínimos Quadrados Generalizados (MQG) com a forma funcional logarítmica. O painel de madeira do tipo aglomerado comportou-se como um substituto ao compensado, e o seu preço influenciou com uma sensibilidade próxima à unitária o preço do compensado no mercado internacional. Com relação ao aumento da renda no mercado internacional, o compensado brasileiro se comportou como um bem inferior.

Palavras-chave: Painéis de madeira; MQG; compensado.
\end{abstract}

\section{Abstract}

Econometric analysis of Brazil's plywood international price. This work analyzes the international price of the Brazilian plywood. Particularly, it estimates the elasticity of the main variables that affect its formation. As methodological base, it was used the method of Generalized Least Squares (GLS) with logarithmic functional form. The particleboard stood out as substitute to plywood, and its price influenced with an almost unitary sensibility the plywood price in international market. Regarding the income increase in the international market, Brazilian plywood stand aside as an inferior good.

Keywords: Wood-based panels; GLS; plywood.

\section{INTRODUÇÃO}

O compensado, um produto com expressiva participação na pauta de exportações de produtos florestais do Brasil, representou em $200824 \%$ do valor exportado da indústria da madeira (SECRETARIA DE COMÉRCIO EXTERIOR - SECEX, 2009).

O Brasil apresenta vantagens competitivas para a indústria de compensado em função do baixo custo do insumo madeira. A alta produtividade das florestas plantadas no Brasil corrobora essa redução de custos da madeira, principal insumo para produção do painel de compensado.

Apesar do baixo custo na produção de madeira, o desempenho das exportações de compensado brasileiro representou apenas $8 \%$ do comércio mundial em 2007 (ORGANIZAÇÃO DAS NAÇÕES UNIDAS PARA AGRICULTURA E ALIMENTAÇÃO (FAO), 2009). Essa baixa representatividade deve-se ao custo Brasil, à falta de planejamento e, recentemente, à grande competitividade da China nesse mercado.

Algumas instituições, como a Associação Brasileira da Indústria de Madeira Processada Mecanicamente (ABIMCI), a Organização Internacional de Madeira Trópica (ITTO) e a Associação Brasileira da Indústria de Painéis de Madeira (ABIPA), exercem um importante papel no fornecimento de informações para o setor. Alguns trabalhos quantitativos que merecem destaque são: Sperandio (1989), Raimundo (2001), Ferreira (1994), Brasil (2002) e Calderon (2005).

Sperandio (1989) e Ferreira (1994) trataram sobre o mercado interno de compensado. Raimundo (2001), Brasil (2002) e Calderon (2005) identificaram as variáveis que afetam a oferta e demanda das exportações brasileiras de painéis de madeira. 
Assim sendo, este trabalho aborda as exportações brasileiras de compensado. Os objetivos específicos foram identificar as elasticidades das principais variáveis que afetam o preço do compensado brasileiro no mercado internacional. As hipóteses esperadas são de uma relação direta entre o preço do compensado com os seus custos de produção e preço de bens substitutos, uma relação indireta com a evolução tecnológica e incerta com a renda, dependendo do caráter de bem inferior ou superior do compensado brasileiro no mercado internacional.

\section{MATERIAL E MÉTODO}

A metodologia foi de acordo com Koutsoyiannis (1978). Segundo o autor, qualquer investigação econométrica pode ser distinguida basicamente em quatro fases: (1) formulação da hipótese sustentada, (2) estimação, (3) avaliação do modelo e (4) avaliação do poder de previsão do modelo. Devido ao objetivo não ser estimar um modelo para previsão, a fase 4 proposta por Koutsoyiannis (1978) foi desconsiderada.

\section{Formulação da hipótese sustentada}

A formação do preço de um produto se dá pela interação de suas curvas de oferta e demanda. Assim, a formação do preço depende das variáveis que afetam a sua demanda e oferta.

De modo geral, demanda e oferta são influenciadas por preço da mercadoria, renda monetária, gosto ou preferência, preços de outras mercadorias relacionadas, tecnologia e custos de produção.

Assim, foram definidas as seguintes variáveis como determinantes na formação do preço pago aos exportadores do compensado brasileiro $\left(P_{e x} C_{t}\right)$ :

- custos de produção $\left(P_{i n} L_{t}\right)$ : foi considerado como proxy dessa variável a evolução do preço internacional da madeira laminada (FAO, 2009). Conforme Gonçalves; Almeida (1998), dos insumos utilizados na fabricação do compensado, é a madeira o que tem o maior peso na composição dos custos industriais, contribuindo com aproximadamente $50 \%$ dos custos totais.

- tecnologia $\left(T_{t}\right)$ : a evolução tecnológica foi medida por uma variável de tendência, conforme sugestão de Gujarati (2000). Segundo o autor, pode ser conveniente supor que a tecnologia seja alguma função do tempo medida cronologicamente.

- renda mundial $\left(R M_{t}\right)$ : calculada a partir do crescimento real do PIB Mundial (IPEA, 2009). Brasil (2002), em um modelo explicativo da demanda de painéis, utilizou como proxy dessa variável as importações mundiais de painéis de madeira.

- $\quad$ preço de bens substitutos: em função da proximidade de uso do compensado com outros painéis de madeira, considerou-se o preço internacional do aglomerado $\left(P_{i n} S_{t}^{A G}\right)$ como um bem substituto (FAO, 2009). Ferreira (1994) e Sperandio (1989) ressaltaram a substitutibilidade do compensado por outros painéis e, entre os painéis com séries históricas disponíveis, o aglomerado é o mais próximo do compensado.

Todas as hipóteses assumidas a princípio foram de acordo com as tabelas 1 e 2 . O modelo teórico do preço internacional do compensado brasileiro pode ser escrito da seguinte forma:

$$
P_{e x} C_{t}=\alpha_{0}+\alpha_{1} P_{i n} L_{t}+\alpha_{2} T_{t}+\alpha_{3} R M_{t}+\alpha_{4} P_{i n} S_{t}^{A G}+\varepsilon_{t}
$$

Em que: $P_{e x} C_{t}=$ preço FOB pago às exportações de compensado do Brasil $\left(\mathrm{US} \$ / \mathrm{m}^{3}\right)$;

$P_{i n} L_{t}=$ preço internacional do laminado $\left(\mathrm{US} \$ / \mathrm{m}^{3}\right)$;

$T_{t}=$ evolução da tecnologia (variável tendência);

$R M_{t}=$ renda mundial ( $\mathrm{n}^{\circ}$ índice);

$P_{i n} S_{t}^{A G}=$ preço internacional do aglomerado $\left(\mathrm{US} \$ / \mathrm{m}^{3}\right)$;

$\varepsilon_{t}=$ termo de erro da equação.

Conforme a tabela 1, é esperada uma relação direta do preço internacional do compensado com o custo de produção e preço internacional do aglomerado. Em relação à evolução da tecnologia, é esperada uma relação indireta. Para a renda, a relação é incerta, podendo ser direta e, assim, indicar o compensado como um bem superior, ou indireta, e sugeri-lo como um bem inferior. 
Devido à falta de um significado prático da constante, a mesma não foi testada. Segundo Hair et al. (2005), o teste $t$ raramente é necessário para os termos de intercepto.

Tabela 1. Hipóteses referentes aos parâmetros.

Table 1. Hypotheses regarding the parameters.

\begin{tabular}{lcc}
\hline Coeficiente & Sinal esperado & Nível de significância considerado \\
\hline$\alpha_{0}$ & - & - \\
$\alpha_{1}$ & $>0$ & \\
$\alpha_{2}$ & $<0$ & $5 \%$ \\
$\alpha_{3}$ & $\neq 0$ & \\
$\alpha_{4}$ & $>0$ & \\
\hline Fonte: Elaborado pelos autores. &
\end{tabular}

Outras hipóteses testadas que são básicas para a obtenção de estimativas confiáveis em um modelo de regressão são a inexistência de problemas de multicolinearidade, heteroscedasticidade, autocorrelação e especificação (Tabela 2).

Tabela 2. Hipóteses do modelo de regressão linear.

Table 2. Hypotheses of the model of linear regression.

\begin{tabular}{lcc}
\hline Esperado & Problema & Nível de significância considerado \\
\hline \multirow{3}{*}{ Ausência } & $\begin{array}{c}{ }^{1} \text { Multicolinearidade } \\
\text { Heteroscedasticidade } \\
\text { Autocorrelação } \\
\text { Especificação }\end{array}$ & - \\
\hline
\end{tabular}

\section{Fonte: Elaborado pelos autores.}

Nota: ${ }^{1}$ Devido à multicolinearidade ser uma característica da amostra, não é feito um "teste da multicolinearidade". Geralmente, sua avaliação é através de regras práticas e valor FIV (Fator de Inflação da Variância). Gujarati (2000) sugere como indício de multicolineariedade valor de FIV superior a 10 .

\section{Estimação}

Os dados utilizados foram séries temporais anuais durante o período de 1961 até 2007 (Tabela 3). O PIB mundial foi coletado em termos reais e os outros valores monetários (preço internacional do compensado, laminado e aglomerado) foram corrigidos através do Índice de Preço ao Consumidor dos Estados Unidos (CPI).

$\mathrm{O}$ preço pago às exportações de compensado brasileiro foi obtido através do quociente entre o seu valor e quantidade exportada. Similarmente, o preço internacional do laminado e aglomerado foi entendido como a razão entre os seus respectivos valores e quantidades mundiais exportadas.

\section{RESULTADOS}

Através do método de Mínimos Quadrados Ordinários (MQO) e optando pela forma funcional logarítmica, devido à simplicidade para serem obtidas as elasticidades diretamente, foi considerada a equação (1) como explicativa do preço pago às exportações do compensado brasileiro.

$$
\begin{array}{llcccc}
\multicolumn{5}{c}{P_{e x} C_{t}=-4,01-0,55 \ln P_{i n} L_{t}-0,06 T_{t}+1,16 \ln R M_{t}+1,61 \ln P_{i n} S_{t}^{A G}} \\
\text { Teste } t & (-1,05) & (-3,01) & (-1,82) & (1,31) & (6,17) \\
\text { FIV } & (5,36) & (4,96) & (318,19) & (335,06) \\
\mathrm{n}=47 & \mathrm{R}^{2} \mathrm{aj}=0,86 \quad \mathrm{~F}=71,93 & d=1,34
\end{array}
$$

Não foi considerada a quantidade exportada pelo Brasil como variável explicativa e tampouco o método de equações simultâneas, pelo motivo da baixa representatividade do país no mercado internacional de compensado e possível caracterização do país como um tomador de preço. Conforme a 
FAO (2009), a representatividade média do Brasil no mercado internacional de compensado no período analisado (1961 a 2007) foi inferior a 5\% da quantidade comercializada.

Tabela 3. Banco de dados utilizados.

Table 3. Used database.

\begin{tabular}{|c|c|c|c|c|c|}
\hline Ano & $P_{e x} C_{t}\left(\mathrm{US} \$ / \mathbf{m}^{3}\right)$ & $P_{i n} L_{t}\left(\mathrm{US} \$ / \mathbf{m}^{3}\right)$ & $P_{i n} S_{t}^{A G}\left(\mathbf{U S} \$ / \mathbf{m}^{3}\right)$ & $\begin{array}{c}R M_{t} \\
\left(n^{0} \text { índice) }\right.\end{array}$ & $\begin{array}{c}T_{t} \\
\text { (tendência) }\end{array}$ \\
\hline 1961 & 806,11 & $1.957,83$ & 413,09 & 100,00 & 1 \\
\hline 1962 & 796,23 & $1.876,40$ & 381,55 & 104,60 & 2 \\
\hline 1963 & 772,10 & $1.792,11$ & 374,60 & 109,83 & 3 \\
\hline 1964 & 729,81 & $1.662,33$ & 377,99 & 116,53 & 4 \\
\hline 1965 & 736,46 & $1.687,78$ & 382,10 & 122,59 & 5 \\
\hline 1966 & 652,97 & $1.504,89$ & 376,82 & 129,09 & 6 \\
\hline 1967 & 803,06 & $1.426,30$ & 369,13 & 134,38 & 7 \\
\hline 1968 & 828,26 & $1.338,92$ & 352,28 & 140,43 & 8 \\
\hline 1969 & 884,54 & $1.377,64$ & 353,88 & 148,85 & 9 \\
\hline 1970 & 740,60 & $1.358,82$ & 356,93 & 153,91 & 10 \\
\hline 1971 & 765,23 & $1.120,31$ & 337,46 & 159,61 & 11 \\
\hline 1972 & 867,02 & $1.166,94$ & 373,47 & 167,11 & 12 \\
\hline 1973 & 808,36 & $1.581,70$ & 411,07 & 176,97 & 13 \\
\hline 1974 & $1.223,00$ & $1.369,52$ & 434,85 & 181,04 & 14 \\
\hline 1975 & 954,09 & $1.405,46$ & 412,83 & 183,57 & 15 \\
\hline 1976 & 907,82 & $1.373,35$ & 397,59 & 193,30 & 16 \\
\hline 1977 & 735,36 & $1.262,42$ & 404,11 & 201,81 & 17 \\
\hline 1978 & 976,85 & $1.264,12$ & 419,78 & 210,08 & 18 \\
\hline 1979 & 992,18 & $1.673,14$ & 453,80 & 218,70 & 19 \\
\hline 1980 & $1.053,50$ & $1.557,16$ & 461,31 & 223,29 & 20 \\
\hline 1981 & 971,21 & $1.167,57$ & 390,59 & 228,20 & 21 \\
\hline 1982 & 854,75 & $1.087,67$ & 354,79 & 230,71 & 22 \\
\hline 1983 & 685,10 & 822,30 & 314,90 & 237,63 & 23 \\
\hline 1984 & 613,72 & 804,90 & 272,09 & 248,80 & 24 \\
\hline 1985 & 541,92 & 803,36 & 269,65 & 258,25 & 25 \\
\hline 1986 & 584,47 & 804,89 & 321,11 & 267,81 & 26 \\
\hline 1987 & 703,91 & 882,66 & 361,66 & 278,25 & 27 \\
\hline 1988 & 659,12 & $1.073,30$ & 341,27 & 291,33 & 28 \\
\hline 1989 & 530,57 & $1.177,22$ & 338,66 & 302,40 & 29 \\
\hline 1990 & 523,61 & $1.000,02$ & 344,17 & 311,17 & 30 \\
\hline 1991 & 585,05 & 993,97 & 330,21 & 316,15 & 31 \\
\hline 1992 & 545,79 & 977,38 & 341,00 & 323,74 & 32 \\
\hline 1993 & 600,55 & 910,71 & 292,42 & 331,51 & 33 \\
\hline 1994 & 564,60 & 972,61 & 310,82 & 344,11 & 34 \\
\hline 1995 & 632,01 & 983,69 & 336,90 & 356,84 & 35 \\
\hline 1996 & 568,40 & 894,25 & 300,27 & 371,47 & 36 \\
\hline 1997 & 556,84 & 850,88 & 261,23 & 387,07 & 37 \\
\hline 1998 & 455,81 & 816,55 & 282,06 & 397,52 & 38 \\
\hline 1999 & 382,09 & 679,17 & 280,81 & 412,63 & 39 \\
\hline 2000 & 327,94 & 677,00 & 231,24 & 432,02 & 40 \\
\hline 2001 & 277,15 & 669,71 & 202,07 & 441,53 & 41 \\
\hline 2002 & 238,73 & 677,35 & 197,15 & 453,89 & 42 \\
\hline 2003 & 278,51 & 750,80 & 241,83 & 470,23 & 43 \\
\hline 2004 & 226,79 & 767,62 & 283,90 & 493,27 & 44 \\
\hline 2005 & 322,95 & 898,73 & 266,04 & 515,47 & 45 \\
\hline 2006 & 233,28 & 942,12 & 243,05 & 541,75 & 46 \\
\hline 2007 & 269,05 & $1.035,31$ & 251,96 & 568,84 & 47 \\
\hline
\end{tabular}

Fonte: FAO (2009) e IPEA (2009). 


\section{Avaliação}

De acordo com a tabela 4, exceto para a variável representante dos custos de produção do compensado (preço do laminado), todos os outros coeficientes mostraram-se com sinal coerente com a teoria econômica e foram conforme o esperado.

Tabela 4. Avaliação dos resultados do modelo com base nos critérios econômico e estatísticos.

Table 4. Evaluation of results of the model based on economical and statistical criteria.

\begin{tabular}{lcccc}
\hline \multirow{2}{*}{ Coeficientes } & \multicolumn{2}{c}{ Sinal } & \multicolumn{2}{c}{ Nível de significância } \\
\cline { 2 - 5 } & Esperado & Encontrado & Esperado & Encontrado \\
\hline$\alpha_{0}$ & $>0$ & & - & - \\
$\alpha_{1}$ & $<0$ & & $\sqrt{ }$ \\
$\alpha_{2}$ & $\neq 0$ & $\sqrt{ }$ & $5 \%$ & $\sqrt{ }$ \\
$\alpha_{3}$ & $\neq 0$ & $\sqrt{ }$ & & $\mathrm{ns}$ \\
$\alpha_{4}$ & $>0$ & $\sqrt{ }$ & $\sqrt{ }$ \\
\hline
\end{tabular}

Fonte: Elaborado pelos autores.

Nota: ns (não significativo).

A incoerência do sinal encontrado deve-se, provavelmente, à variável preço internacional do laminado não ser uma boa proxy dos custos de produção do compensado nacional. O custo da madeira para a indústria de base florestal nacional é bem inferior à média mundial, devido, basicamente, à alta produtividade das florestas plantadas no Brasil e ao grande estoque e baixo preço da madeira tropical. Possivelmente, o preço internacional do laminado não reflete o custo das empresas nacionais de compensado para obtenção dessa matéria-prima. Em geral, as empresas de compensados nacionais são verticalizadas (produzem seu próprio laminado) e importações desse produto são desprezíveis.

Os resultados estatísticos sugerem a presença de multicolinearidade, sendo obtido um valor FIV bem acima de 10 para as variáveis tecnologia e renda. O grau de correlação entre essas variáveis no período analisado é de 0,99 , indicando ser praticamente impossível estimar com confiança o efeito separado de cada variável em um modelo de regressão.

A avaliação da heteroscedasticidade, autocorrelação e especificação foi através do teste BPG de Breusch e Pagan (1979), estatística $d$ de Durbin e Watson e teste RESET de Ramsey (1969). De acordo com esses testes, não foram detectados problemas de heteroscedasticidade e especificação. Já a estatística $d$ não foi decisiva para determinar a autocorrelação. Como alternativa, foi aplicado o teste de Geary (1970), que não rejeitou a hipótese de autocorrelação com uma probabilidade de erro inferior a 5\% de chance (Tabela 5).

Tabela 5. Avaliação dos resultados do modelo com base no critério econométrico.

Table 5. Evaluation of results of the model based on the econometric criterion.

\begin{tabular}{lcc}
\hline Problema & Esperado & Encontrado \\
\hline Multicolinearidade & & Presença \\
Heteroscedasticidade & Ausência & $\sqrt{ }$ \\
Autocorrelação & & Presença \\
Especificação & & $\sqrt{ }$ \\
\hline Fonte: Elaborado pelos autores. & &
\end{tabular}

\section{Reestimação}

Conforme Gujarati (2000), na prática nunca temos certeza de que o modelo adotado para o teste empírico é a "a verdade, somente a verdade e nada mais que a verdade". Dessa forma, o autor sugere a readequação do modelo em casos em que os resultados não sejam satisfatórios.

O problema de multicolinearidade foi corrigido através da exclusão das variáveis preço internacional do laminado e tecnologia (variável de tendência). A primeira por apresentar o sinal errado e, aparentemente, não ser uma boa proxy dos custos de produção. A segunda por ser altamente correlacionada com a renda mundial e, teoricamente, ser bastante frágil. Naturalmente, é questionável a consideração de uma evolução tecnológica como gradualmente crescente ao longo do tempo. 
As estimativas do modelo após a correção dos problemas de multicolineariedade foram de acordo com a equação (2).

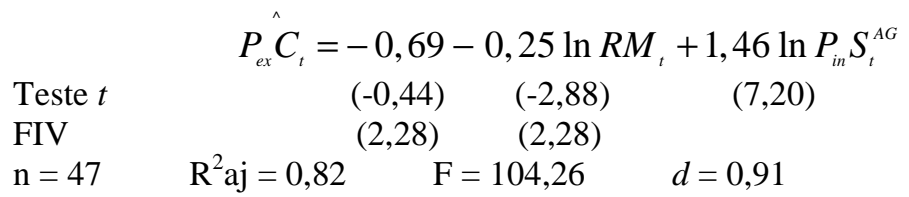

Com a exclusão das variáveis preço internacional do laminado e tecnologia, o problema da autocorrelação foi agravado, podendo agora ser detectado pela estatística $d$ (valor 0,91 , inferior ao limite inferior tabelado para o nível de significância de 5\%). Para corrigir o problema da autocorrelação, foi aplicado o método dos Mínimos Quadrados Generalizados (MQG) (equação 3).

$$
P_{e x} C_{t}=1,7-0,49 \ln R M_{t}+0,93 \ln P_{i n} S_{t}^{A G}
$$

$$
\begin{array}{lrrr}
\text { Teste } t & (1,97) & (-3,83) & (3,90) \\
\text { FIV } & (1,57) & (1,57) & \\
\mathrm{n}=46 & \mathrm{R}^{2} \mathrm{aj}=0,62 & \mathrm{~F}=37,73 & d=2,18
\end{array}
$$

\section{Reavaliação}

Conforme a tabela 6 , todos os coeficientes estimados na equação 3 mostraram-se com o sinal coerente e foram estatisticamente significativos. Exceto para constante (significativa ao nível de 5\%), todos os outros coeficientes foram altamente significativos e estimados com uma probabilidade de erro inferior a $1 \%$ de chance.

Tabela 6. Avaliação dos resultados do modelo com base nos critérios econômico e estatísticos.

Table 6. Evaluation of results of the model based on the economical and statistical criteria.

\begin{tabular}{lcccc}
\hline \multirow{2}{*}{ Coeficientes } & \multicolumn{2}{c}{ Sinal } & \multicolumn{2}{c}{ Nível de significância } \\
\cline { 2 - 5 } & Esperado & Encontrado & Esperado & Encontrado \\
\hline$\alpha_{0}$ & $\neq 0$ & $\sqrt{ }$ & - & - \\
$\alpha_{3}$ & $>0$ & $\sqrt{ }$ & $5 \%$ & $\sqrt{ }$ \\
$\alpha_{4}$ & & & & \\
\hline
\end{tabular}

Fonte: Elaborado pelos autores.

Os testes econométricos foram satisfatórios, os valores de FIV sugeriram a ausência de multicolineariedade e a nova estatística $d$ referenda a correção do problema de autocorrelação (Tabela 7).

Segundo Gujarati (2000), a estatística $d$ pode não ser adequada para testar a presença de autocorrelação nos dados que tenham sido ajustados por MQG. Nesse caso, o autor recomenda a aplicação do teste de Geary (1970). O teste de Geary (1970) rejeitou o problema de autocorrelação ao nível de significância de 5\%.

Tabela 7. Avaliação dos resultados do modelo com base no critério econométrico.

Table 7. Evaluation of results of the model based on the econometric criterion.

\begin{tabular}{lcc}
\hline Problema & Esperado & Encontrado \\
\hline Multicolinearidade & & $\sqrt{ }$ \\
Heteroscedasticidade & Ausência & $\sqrt{ }$ \\
Autocorrelação & & $\sqrt{ }$ \\
Especificação & & $\sqrt{ }$ \\
\hline Fonte: Elaborado pelos autores.
\end{tabular}

A redução do $\mathrm{R}^{2}$ aj entre os modelos com a presença de autocorrelação e estimado por MQG (equações 2 e 3) é esperada, visto que a variância do resíduo estimada enganadamente por MQO provavelmente subestime o seu verdadeiro valor e, consequentemente, superestime o valor do $R^{2}$ (GUJARATI, 2000). 


\section{DISCUSSÃO}

Foi possível explicar o preço pago às exportações do compensado brasileiro apenas com variáveis relacionadas à demanda. Esse resultado sugere a pouca influência da oferta na formação do preço externo do compensado nacional, reforça a hipótese do Brasil como tomador de preço no mercado internacional e credibiliza a não aplicação do método de equações simultâneas.

Os resultados para o efeito do aglomerado indicaram uma elasticidade próxima à unitária, na qual um aumento de $1 \%$ no preço internacional do aglomerado leva a um aumento de $0,93 \%$ no preço pago às exportações do compensado brasileiro. Naturalmente, é esperado que o aumento do preço internacional do aglomerado leve a um aumento da demanda externa pelo compensado nacional e, consequentemente, a uma pressão no seu preço.

Em relação à direção do sinal encontrado e sugestão de uma relação de substituição entre o compensado e o aglomerado, destaca-se a tendência observada nas últimas décadas e apontada para o futuro em relação à utilização de toras de pequeno diâmetro e minimização na geração de resíduos. Esses fatores vêm levando a uma perda de mercado e competitividade do compensado no mercado mundial.

De acordo com os dados da FAO (2009), no período de 1961 a 2007, a produção mundial de compensado cresceu a uma taxa de $6 \%$ ao ano, enquanto que a de aglomerado foi quase o dobro, alcançando um valor de $11 \%$ ao ano no mesmo período.

Os resultados encontrados por Brasil (2002) utilizando o modelo da elasticidade de substituição também indicaram uma relação de substituição entre o compensado e o aglomerado para o mercado externo. Conforme o autor, para um aumento de $1 \%$ no preço do compensado, espera-se o aumento de $0,72 \%$ nas exportações de painéis de partícula.

A elasticidade renda encontrada sugeriu o compensado como um bem inferior, em que o aumento de $1 \%$ na renda mundial leva a uma queda de $0,45 \%$ no preço externo do compensado nacional. Esses resultados estiveram em desacordo com Brasil (2002), o qual sugeriu o compensado como um bem superior em uma magnitude altamente elástica. Conforme o autor, um aumento de $1 \%$ na renda mundial leva ao aumento de $1,6 \%$ na demanda de compensado. Destaca-se que a variável utilizada por Brasil (2002) na estimativa da elasticidade renda não foi a mesma do presente trabalho - tratou-se de proxy medida através das importações mundiais de painéis de madeira.

Os resultados encontrados referentes ao efeito da renda mundial no preço pago às exportações do compensado brasileiro sugerem que um aumento da renda mundial leva a uma mudança de consumo em direção de bens produzidos com outros materiais (metal, plástico e vidro, entre outros) e/ou para painéis mais modernos, como o OSB ou MDF.

\section{CONCLUSÕES}

- O preço externo do compensado brasileiro pode ser explicado apenas por variáveis relacionadas à demanda (renda mundial e preço internacional do aglomerado), sugerindo, portanto, uma baixa influência da oferta na formação desse preço e indicando o Brasil como um tomador de preço no mercado internacional de compensado.

- O aglomerado apresentou-se como um bem substituto ao compensado no mercado internacional.

- Os resultados sugeriram o compensando como um bem inferior no mercado internacional e esteve em desacordo com o encontrado em outra pesquisa.

- Recomendam-se outras pesquisas quantitativas que avaliem o efeito da renda e de outras variáveis para o mercado de compensado.

\section{REFERÊNCIAS}

BRASIL, A. A. As exportações brasileiras de painéis de madeira. Dissertação (Mestrado em Ciências Florestais) - Setor de Ciências Agrárias, Universidade Federal do Paraná, Curitiba, PR. 2002. 74 p.

BREUSCH, T.; PAGAN, A. A simple test for heteroscedasticity and random coefficient variation. Econometrica, n. 47, p. 1287 - 1294, 1979. 
CALDERON, R. A. Funções de oferta e demanda de exportações para manufaturados de madeira. Dissertação (Mestrado em Ciências Florestais) - Universidade de Brasília, Brasília, DF. 2005. 56 p.

FAO. Food and Agriculture Organization of the United Nations. Disponível em: <http://faostat.fao.org/site/626/default.aspx\#ancor>. Acesso em: 28/02/2009.

FERREIRA, A. M. Análise da demanda por compensados no Brasil. Dissertação (Mestrado em Ciências Florestais) - Setor de Ciências Agrárias, Universidade Federal do Paraná, Curitiba, PR. 1994. 64 p.

GEARY, R. C. Relative Efficiency of Count of Sign Changes for Assessing Residual Autoregression in Least Squares Regression. Biometrika, n. 57, p. 123 - 127, 1970.

GUJARATI, D. N. Econometria Básica. 3. ed., São Paulo: Makron Books. 846 p. 2000.

HAIR JR., J. F.; ANDERSON, R. E.; TATHAM, R. L.; BLACK, W. C. Análise multivariada de dados. 5. ed., Porto Alegre: Bookman. 593 pp. 2005.

IPEA. Disponível em: <http://www.ipeadata.gov.br>. Acesso em: 28/02/2009.

KOUTSOYIANNIS, A. Theory of Econometrics. 2. ed. New Jersey: Barnes\&Noble Books, 1978. 683 p.

RAMSEY, J. B. Tests for Specification Error in Classical Linear Least Squares Regression Analysis. Journal of the Royal Statistical Society, série B, v. 31, p. 350 - 371, 1969.

SECEX. Secretaria de Comércio Exterior. Disponível em: <http://aliceweb.desenvolvimento.gov.br/>. Acesso em: 28/02/2009.

SPERANDIO, J. P. Estudo econométrico da demanda e oferta de compensado para o Estado do Paraná. Dissertação (Mestrado em Ciências Florestais) - Setor de Ciências Agrárias, Universidade Federal do Paraná, Curitiba, PR. 1989. 110 p.

RAIMUNDO, Y. M. Análise das exportações brasileiras de madeira serrada e painéis à base de madeira no período de 1961 a 1999. Dissertação (Mestrado em Economia Aplicada) - Escola Superior de Agricultura Luiz de Queiroz, Piracicaba, SP. 2001. 141 p. 